\title{
Review of the Multidisciplinary Voice Clinic in a District General Hospital of the United Kingdom
}

\author{
Louise Marie Evans, ${ }^{1}$ Parry Haf Beca, ${ }^{1}$ Helen Patterson, ${ }^{1}$ Alagar Chandra Mohan ${ }^{1}$
}

\section{$\underline{\text { Introduction }}$}

\section{ABSTRACT}

The Voice Clinic is a multidisciplinary specialist voice clinic. Our aim is to review patient demographics, subjective symptoms both at presentation and 16 months post clinic and patient satisfaction.

\section{Methods}

A retrospective review of all pre treatment voice clinic questionnaires for all new patients who attended the voice clinic over a 3-month period. A telephone interview was conducted 16 months after first presentation to determine patient satisfaction and whether patient's symptoms had improved.

$\underline{\text { Results }}$

A total of 25 patients who had completed a pre clinic questionnaire were identified, 17/25 completed the follow up interview. $88 \%$ of patients reported symptoms of $\geq 6$ months duration. $41 \%$ underwent Speech and Language Therapy (SALT), 24\% had medical management, 6\% surgery, and 30\% were given advice only. On a severity scale of $1-10$ (10 most severe) $40 \%$ rated their voice severity to be $\geq 5 / 10$ at presentation, 16 months later this was $11 \%$ of patients. $56 \%$ rated the impact of their voice on their life to be $\geq 5 / 10$ at presentation, 16 months later this was $6 \%$.

\section{Conclusion}

Although limited the review shows that for the majority of patients their symptoms and quality of life improved after review at the multidisciplinary clinic.

$\underline{\text { Keywords }}$

Voice; Speech; Patient Satisfaction; Quality of Life

$\mathrm{T}$ The Voice Clinic aims to provide patients with a multidisciplinary specialist approach to the evaluation of voice disorders in a District General Hospital. Patients are referred via the GP or the general ENT clinic if a specialist voice assessment is required.

Prior to the appointment whilst in the waiting room all patients are given a pre treatment voice clinic questionnaire which asks questions regarding their symptoms, their severity, the impact these symptoms may have on their lives, their lifestyle and their past medical history. Each patient is given a twenty-minute appointment and is seen by both a Speech and Language

1 - Wrexham Maelor Hospital, Wales, UK

\section{Corresponding author:}

Dr Louise Marie Evans

email: louiseevans_5@hotmail.co.uk
Therapist and a Consultant Otolaryngologist. After discussion of the presenting complaint and the context of the symptoms a discussion is had exploring the impact this has on the patient's lives.

The larynx is examined using Stroboscopy (Fig. 1) and the voice analysis is recorded and viewed by the patient and the pathology or indeed the normal examination explained. This provides immediate feedback and often reassurance for the patient. The aim of this study is to review patient demographics, subjective symptoms both at presentation and 16 months post clinic and patient satisfaction.

\section{Materials and Methods}

A retrospective review of the pre treatment voice clinic questionnaires for all new patients who attended the 


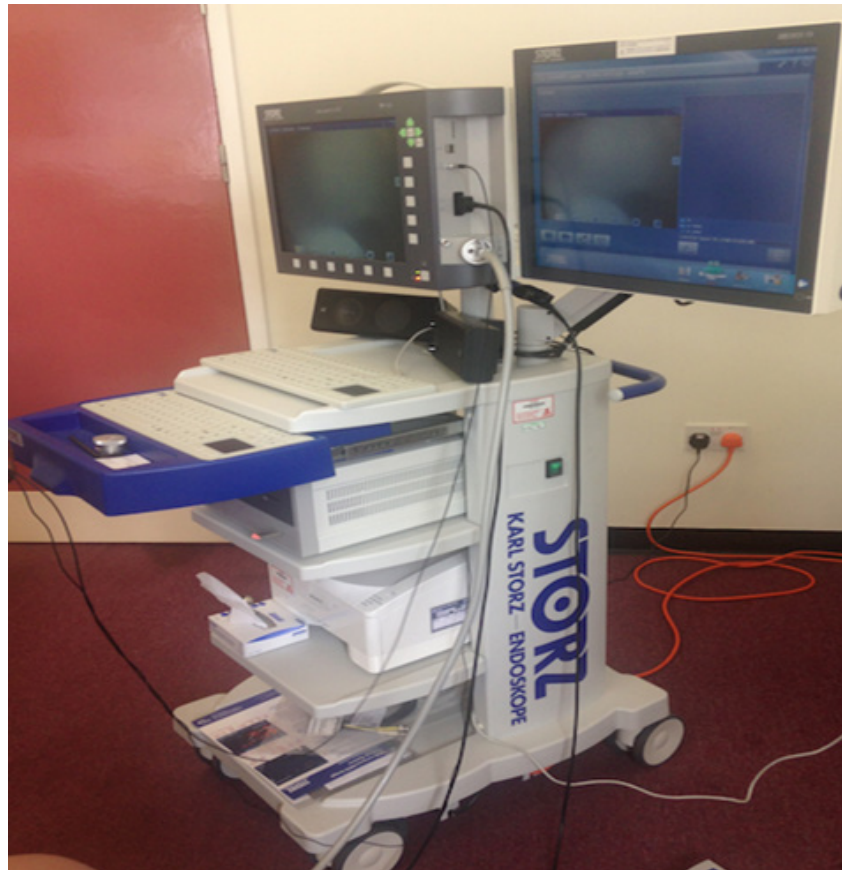

Fig.1 Stroboscopy equipment

voice clinic over a 3-month period was conducted and patient demographics and subjective symptoms noted. A telephone interview was conducted 16 months after the first clinic appointment to determine patient satisfaction, the severity of symptoms and the impact of the symptoms on the patients' lives.

\section{Results}

25 patients who had completed a pre-clinic questionnaire were identified, 17/25 completed the follow up interview. The pre treatment patients $\mathrm{F}$ : $\mathrm{M}$ ratio was $3: 1$ and $48 \%$ of patients were $>60$ years old. $88 \%$ of patients reported symptoms of $\geq 6$ months duration. $41 \%$ underwent SALT, 24\% medical management, $6 \%$ surgery, and $30 \%$ advice only. On a severity scale of 1- 10 (10 most severe) $40 \%$ rated their voice severity to be $\geq 5 / 10$ (Fig.2), 16 months later this was $11 \%$. 56\% rated the impact of their voice on their life to be $\geq 5 / 10$, 16 months later this was $6 \%$ (Fig. 3). The most common symptom (excluding hoarseness) was a voice that fades, changes in pitch and inability to sing, on follow up the

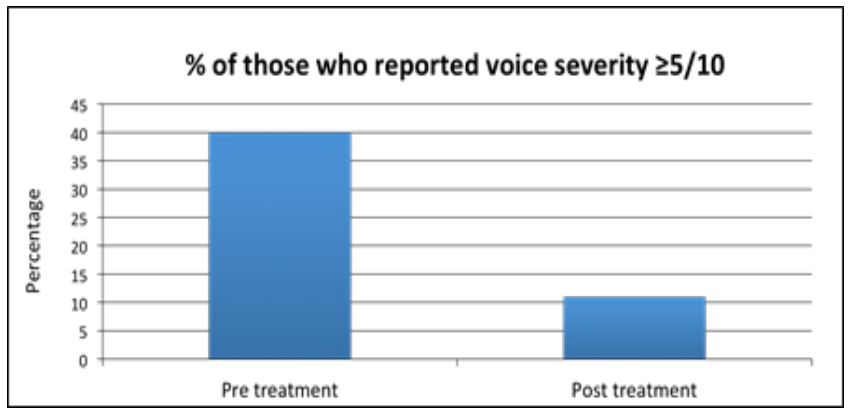

Fig.2 Percentage of those who reported voice severity $\geq 5 / 10$, pre and post treatment

most common symptom was postnasal drip. $76 \%$ of patients felt that the clinic was good or very good and $88 \%$ felt that their concerns were addressed.

\section{Discussion}

A review of the voice clinic has not been conducted before. Although a small study, limited by size it has shown the specialist clinic to be largely successful with good patient satisfaction scores. It is felt in the literature that access to specialist consultant led served allows for more rapid and appropriate decision making, improved outcome and fulfills patient expectations for access to skilled clinicians. ${ }^{1}$ Such consultant led clinics can also benefit the training of more junior doctors. The Voice Clinic is used to deliver one to one training by the Consultant for the Specialist Registrar for which there has been very good feedback from the trainees.

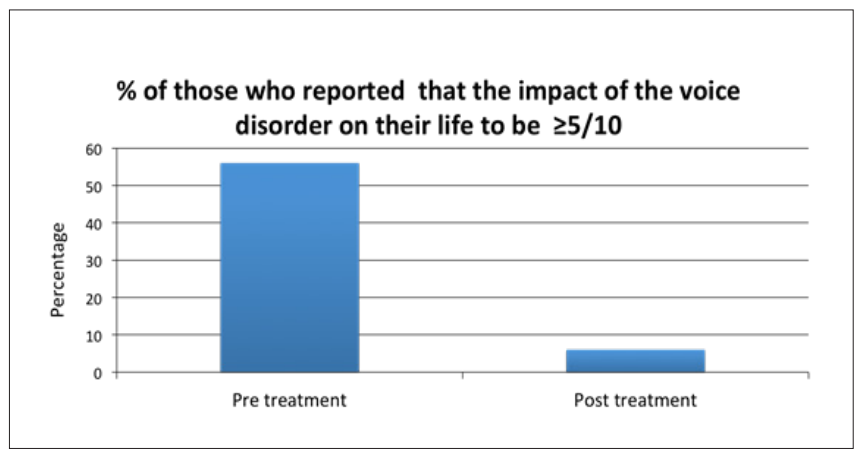

Fig.3 Percentage of those who reported that the impact of the voice disorder on their life to $b e \geq 5 / 10$, pre and post treatment 


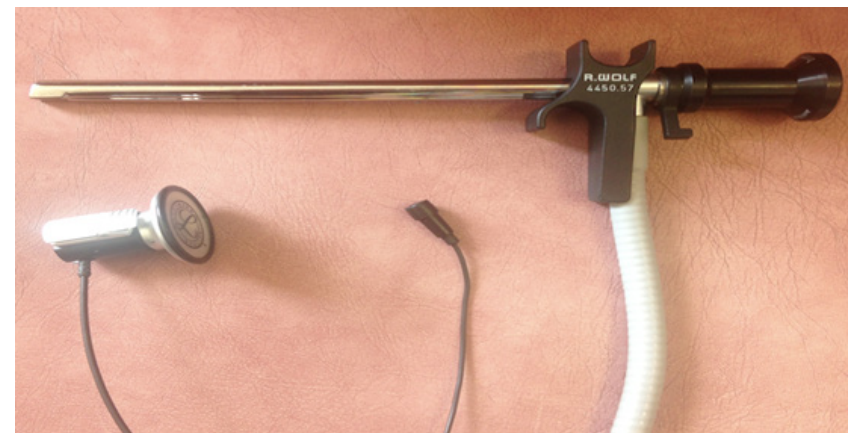

Fig.4 Rigid scope and microphone

The voice clinic as a specialist clinic allows the concentration of resources and expertise so that patients have a multidisciplinary approach to the treatment plan. The importance of the team approach cannot be over emphasised as both the examination and the interpretation of the data collected for each patient requires multi-disciplinary input to arrive at both a diagnosis and plan for treatment (Fig. 4). Cognitive Behavior Therapy (CBT) is a very well evidenced therapy and recommended by The National Institute for Health and Care Excellence (NICE) as the treatment of choice for mental health difficulties and medically unexplained symptoms. ${ }^{2}$ The benefits of CBT for patients with psychological voice disorders such as functional dysphonia has been discussed and a few studies have shown that it can be clinically effective in the treatment of functional dysphonia. ${ }^{2,3}$ This is an area that we would like to explore further as we feel that the clinic may benefit from some specialist psychological input.

Giving the opportunity for patients to self-report symptoms using the questionnaire is a vital tool as it allows the clinician to gain as much information as possible on the problem. The questionnaire gives the opportunity for the patient to discuss how their voice is affecting their life and their voice severity for the majority of the time as their voice on the day of the consultation may not be representative of their voice on a day-to-day basis. ${ }^{4}$ Also unless patients are satisfied with their own voice, we cannot claim that the clinic or indeed or management of the patient is successful and therefore self-reporting scales allow us to compare the effect of intervention. ${ }^{4}$ Such reporting is also vital in the current NHS climate, as we must monitor our service to show it is beneficial to patients and explore how improvements can be made to maximise the effectiveness of the service.

\section{Conclusion}

Although limited the review shows that for the majority of patients their symptoms and quality of life improved after review at the multidisciplinary clinic. The clinic allows direct audiovisual investigation of the voice in a teaching environment. The multidisciplinary approach allows SALT to have direct input into diagnosis and management of patients. The input of a psychologist to assist us in exploring causative factors of voice disorders in some patients we feel may also benefit patients and improve access to treatments such as CBT.

\section{References}

1. Academy of Medical Royal Colleges (Jan 2012). The Benefits Of Consultant- Delivered Care. http://www.aomrc.org.uk/doc view/9450-the-benefits-of-consultant-delivered-care

2. Miller $\mathrm{T}$, Deary $\mathrm{V}$, Patterson J. Improving access to psychological therapies in voice disorders: a cognitive behavioural therapy model. Curr Opin Otolaryngol Head Neck Surg. 2014;22(3):201-5

3. Daniilidou P, Carding P, Wilson J, Drinnan M, Deary V. Cognitive behavioral therapy for functional dysphonia: a pilot study. Ann Otol Rhinol Laryngol. 2007;116(10):717-22

4. Carding PN, Wilson JA, MacKenzie K, Deary IJ. Measuring voice outcomes: state of the science review The Journal of Laryngology \& Otology 2009; 123: 823-829. 